\title{
Lattice phase shifts and mixing angles for an arbitrary number of coupled channels
}

\author{
Lukas Bovermann $^{1 \star}$, Evgeny Epelbaum ${ }^{1}$, Hermann Krebs $^{1}$ and Dean Lee ${ }^{2}$
}

1 Ruhr-Universität Bochum, Fakultät für Physik und Astronomie, Institut für Theoretische Physik II, D-44780 Bochum, Germany

2 Facility for Rare Isotope Beams and Department of Physics and Astronomy, Michigan State University, MI 48824, USA

$\star$ lukas.bovermann@rub.de

$$
\begin{array}{r}
\text { Proceedings for the 24th edition of European Few B } \\
\text { Surrey, UK, 2-6 September } 2019 \\
\text { doi:10.21468/SciPostPhysProc.3 }
\end{array}
$$

\begin{abstract}
We present a lattice method for determining scattering phase shifts and mixing angles for the case of an arbitrary number of coupled channels. The proposed method combines a spherical wall boundary condition and a channel-mixing auxiliary potential to extract the full-rank S-matrix from the radial wave functions. We consider the scattering problem of two spin-1 bosons interacting with a test potential involving up to four coupled channels. For this benchmark system, the phase shifts and mixing angles are shown to agree on the lattice and in the continuum. Our method should allow to extend previous two-channel nuclear lattice EFT simulations to mixing of more than two partial waves.
\end{abstract}

(c) () Copyright L. Bovermann et al.

This work is licensed under the Creative Commons

Attribution 4.0 International License.

Published by the SciPost Foundation.
Received 15-10-2019

Accepted 11-12-2019

Published 27-02-2020

doi:10.21468/SciPostPhysProc.3.052

\section{Introduction}

In nuclear and particle physics, lattice simulations can be applied to many-body problems for which continuum methods would be computationally too expensive. For example, they allow to calculate the hadron spectrum in the non-perturbative domain of QCD [1]. Scattering processes involving few hadrons can also be considered in the lattice QCD framework by relating the infinite-volume S-matrix to the finite-volume energy spectrum. This can be achieved using Lüscher's method [2-4], which has already been generalized to an arbitrary number of coupled scattering channels [5-9].

Simulating many-nucleon systems with lattice QCD is not yet possible due to the large computational costs. Instead, it is more efficient to employ a lattice version of chiral effective field theory (EFT) for such systems. This lattice EFT has been used to calculate bound states of several light and medium-mass nuclei in Refs. [10-14]. Moreover, it has also been applied to nucleon-nucleon, nucleon-nucleus and nucleus-nucleus scattering [10,15-18]. 
Unfortunately, Lüscher's method is not suitable for scattering of heavier nuclei because the finite-volume scattering energies for these nuclei are very small and thus cannot be determined with the required accuracy in Monte Carlo simulations [19]. In order to solve this problem, the adiabatic projection method [20] has been used in Refs. $[15,16]$ to compute an effective nucleus-nucleus Hamiltonian called the adiabatic Hamiltonian. Since the adiabatic Hamiltonian only depends on the distance between the two nuclear clusters, one can extract phase shifts from this Hamiltonian using spherical wall boundary conditions [21,22].

So far, the spherical wall method has only been applied to at most two coupled scattering channels. Because three or more coupled partial waves often appear in nuclear reactions, we develop the (non-trivial) generalization of the two-channel method from Ref. [19] to an arbitrary number of coupled channels in this article.

\section{Benchmark system}

As a concrete example, we consider scattering of two spin-1 bosons with the approximate deuteron mass $m_{1}=m_{2}=2 m_{\mathrm{N}}$ where $m_{\mathrm{N}}=938.92 \mathrm{MeV}$ :

$$
H=\frac{p_{1}^{2}}{2 m_{1}}+\frac{p_{2}^{2}}{2 m_{2}}+V\left(\vec{r}_{1}-\vec{r}_{2}\right)
$$

Here, $p_{1}$ and $p_{2}$ denote the momenta of the two particles, and the potential $V$ only depends on the difference between their positions $\vec{r}_{1}$ and $\vec{r}_{2}$. For spin- $1 / 2$ fermions, a toy-model potential already exists in Refs. [19,22]:

$$
V_{\text {fermion }}(\vec{r})=C\left(1+\frac{3\left(\vec{r} \cdot \vec{\sigma}_{1}\right)\left(\vec{r} \cdot \vec{\sigma}_{2}\right)-\left(\vec{\sigma}_{1} \cdot \vec{\sigma}_{2}\right) r^{2}}{r_{0}^{2}}\right) e^{-r^{2} /\left(2 r_{0}^{2}\right)}
$$

Since the tensor term $3\left(\vec{r} \cdot \vec{\sigma}_{1}\right)\left(\vec{r} \cdot \vec{\sigma}_{2}\right)-\left(\vec{\sigma}_{1} \cdot \vec{\sigma}_{2}\right) r^{2}$ also appears in the one-pion exchange potential in chiral EFT, one can qualitatively interpret Eq. (2) as a nucleon-nucleon interaction and choose the parameters

$$
C=-2 \mathrm{MeV}, \quad r_{0}=3.95 \mathrm{fm}
$$

motivated by typical scales of nuclear systems. On the other hand, Eq. (2) is also similar to a dipole-dipole interaction like

$$
V_{\text {dipole }}(\vec{r}) \propto-\frac{1}{4 \pi r^{3}}\left(3\left(\hat{r} \cdot \vec{\mu}_{1}\right)\left(\hat{r} \cdot \vec{\mu}_{2}\right)-\left(\vec{\mu}_{1} \cdot \vec{\mu}_{2}\right)\right)
$$

in atomic or molecular physics [22]. Moreover, Eq. (2) can be transformed into a test potential for spin- 1 bosons if the Pauli matrices $\vec{\sigma}_{1}, \vec{\sigma}_{2}$ for the two particles are replaced by the spin- 1 matrices $\vec{s}_{1}, \vec{s}_{2}$ :

$$
V(\vec{r})=C\left(1+\frac{3\left(\vec{r} \cdot \vec{s}_{1}\right)\left(\vec{r} \cdot \vec{s}_{2}\right)-\left(\vec{s}_{1} \cdot \vec{s}_{2}\right) r^{2}}{r_{0}^{2}}\right) e^{-r^{2} /\left(2 r_{0}^{2}\right)}
$$

Projecting the potential in Eq. (5) onto partial waves yields up to four coupled scattering channels. 


\section{Lattice method}

\subsection{Calculational setup}

We introduce a cubic lattice with the spacing $a=1.97 \mathrm{fm}$ and the length $L=35 a$, and define orthonormal lattice states $|\vec{r}\rangle$ with

$$
r_{1}, r_{2}, r_{3}=0, \ldots,(L-1) a=0, \ldots,(L-1) \text { l.u., }
$$

where "l.u." denotes dimensionless lattice units, which are used throughout this section. Quantities in lattice units must be multiplied by an appropriate power of the lattice spacing $a$ to obtain their physical values. Moreover, we impose periodic boundary conditions in each direction:

$$
|\vec{r}\rangle=\left|\vec{r}+L \hat{e}_{1}\right\rangle=\left|\vec{r}+L \hat{e}_{2}\right\rangle=\left|\vec{r}+L \hat{e}_{3}\right\rangle .
$$

In the center-of-mass system (CMS), the Hamiltonian can be written as

$$
\begin{aligned}
H|\vec{r}\rangle= & \frac{49}{12 \mu}|\vec{r}\rangle-\frac{3}{4 \mu} \sum_{i=1}^{3}\left(\left|\vec{r}+\hat{e}_{i}\right\rangle+\left|\vec{r}-\hat{e}_{i}\right\rangle\right)+\frac{3}{40 \mu} \sum_{i=1}^{3}\left(\left|\vec{r}+2 \hat{e}_{i}\right\rangle+\left|\vec{r}-2 \hat{e}_{i}\right\rangle\right) \\
& -\frac{1}{180 \mu} \sum_{i=1}^{3}\left(\left|\vec{r}+3 \hat{e}_{i}\right\rangle+\left|\vec{r}-3 \hat{e}_{i}\right\rangle\right)+V(\vec{r})|\vec{r}\rangle
\end{aligned}
$$

with the reduced mass $\mu=m_{1} m_{2} /\left(m_{1}+m_{2}\right)$. The derivative in the free term has been discretized using the $O\left(a^{4}\right)$-improved lattice dispersion relation [22].

\subsection{Projection onto partial waves}

Following Ref. [19], we define radial states for a partial wave ${ }^{2 s+1} l_{j}$ :

$$
|R\rangle_{s, l, j}=\sum_{\vec{r}} \sum_{l_{z}, s_{z}} \sum_{s_{1, z}} \sum_{s_{2, z}} C_{0, l_{z}, s_{z}}^{j, l} C_{s_{z}, s_{1, z}, s_{2, z}}^{s, 1,1} Y_{l, l_{z}}(\hat{r}) \delta_{r, R}|\vec{r}\rangle \otimes\left|s_{1, z}, s_{2, z}\right\rangle,
$$

where the first sum runs over all lattice sites with a certain radial position (due to $\delta_{r, R}$ ). The Clebsch-Gordan coefficients for the spin-orbit and spin-spin couplings are given by $C_{0, l_{z}, s_{z}}^{j, l, s}$ and $C_{s_{z}, s_{1, z}, s_{2, z}}^{s, 1,1}$, respectively, and $Y_{l, l_{z}}$ denotes the spherical harmonics. For $n$ coupled channels with

$$
|R\rangle_{\alpha}:=|R\rangle_{s_{\alpha}, l_{\alpha}, j_{\alpha}},
$$

for $\alpha=1, \ldots, n$, the Hamiltonian can be projected onto the normalized radial states like

$$
\left[H_{R}\left(R_{1}, R_{2}\right)\right]_{\alpha \beta}=\sum_{\alpha^{\prime}, \beta^{\prime}=1}^{n}\left[N^{-1 / 2}\left(R_{1}\right)\right]_{\alpha \alpha^{\prime} \alpha^{\prime}}\left\langle R_{1}|H| R_{2}\right\rangle_{\beta^{\prime}}\left[N^{-1 / 2}\left(R_{2}\right)\right]_{\beta^{\prime} \beta},
$$

where $N^{-1 / 2}$ is the inverse square root of the norm matrix

$$
[N(R)]_{\alpha \alpha^{\prime}}={ }_{\alpha}\langle R \mid R\rangle_{\alpha^{\prime}} .
$$

By computing the eigenvectors $|\psi\rangle$ of the radial Hamiltonian $H_{R}$, one can then obtain the wave function

$$
\psi_{\alpha}(R)=\sum_{\alpha^{\prime}=1}^{n}\left[N^{-1 / 2}(R)\right]_{\alpha \alpha^{\prime} \alpha^{\prime}}\langle R \mid \psi\rangle
$$

in the $\alpha$-th scattering channel. 


\subsection{Auxiliary potentials}

In order to yield accurate results on the lattice, two additional potential terms have to be introduced. The first of these auxiliary potentials is the spherical wall potential that avoids artifacts caused by the periodic boundary conditions. It can be added by replacing

$$
V(\vec{r}) \rightarrow V(\vec{r})+\Lambda \theta\left(r-R_{W}\right),
$$

where $\theta$ denotes the Heaviside function, $R_{W}$ is the wall radius and $\Lambda$ has a large positive value [22]. The second auxiliary potential is the so-called mixing potential, which will be necessary to obtain the full-rank S-matrix [19]. For constructing the $n$-channel S-matrix, one needs $n$ linearly independent solutions of the Schrödinger equation in each channel. This corresponds to a wave function vector with $n^{2}$ components:

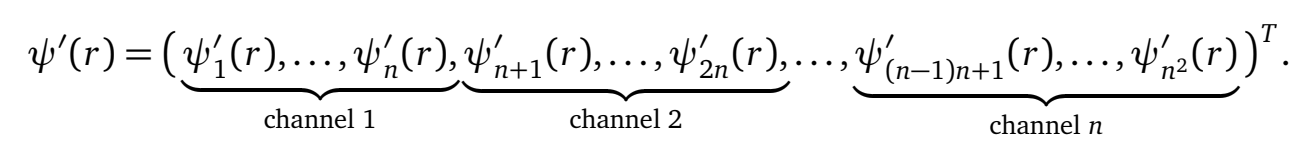

Accordingly, the radial Hamiltonian must be extended to an $n^{2} \times n^{2}$ matrix:

$$
\left[H_{R}^{\prime}\right]_{\alpha^{\prime}+(\alpha-1) n, \beta^{\prime}+(\beta-1) n}=\left[H_{R}\right]_{\alpha, \beta} \delta_{\alpha^{\prime}, \beta^{\prime}},
$$

for $\alpha, \alpha^{\prime}, \beta, \beta^{\prime}=1, \ldots, n$. Now the mixing potential can be added to change the boundary conditions for the different solutions:

$$
H_{R}^{\prime} \rightarrow H_{R}^{\prime}+U_{0} \delta_{r, R_{M}} U_{M}
$$

where $U_{0}$ is a real coefficient and $U_{M}$ is a channel-mixing matrix. The shape of the mixing potential is a $\delta$-peak located close to the spherical wall $\left(R_{M} \lesssim R_{W}\right)$ and outside the range of the test potential. As explained in Ref. [23], the matrix $U_{M}$ can be chosen as

$$
\left[U_{M}\right]_{\alpha^{\prime}+(\alpha-1) n, \beta^{\prime}+(\beta-1) n}=\left(1-\delta_{\alpha, \beta}\right)\left(1-\delta_{\alpha^{\prime}, \beta^{\prime}}-2 \delta_{\alpha, \alpha^{\prime}} \delta_{\beta, \beta^{\prime}}\right),
$$

or explicitly in the case of $n=3$,

$$
U_{M}=\left(\begin{array}{rrr|rrr|rrr}
0 & 0 & 0 & 0 & -1 & 1 & 0 & 1 & -1 \\
0 & 0 & 0 & 1 & 0 & 1 & 1 & 0 & 1 \\
0 & 0 & 0 & 1 & 1 & 0 & 1 & 1 & 0 \\
\hline 0 & 1 & 1 & 0 & 0 & 0 & 0 & 1 & 1 \\
-1 & 0 & 1 & 0 & 0 & 0 & 1 & 0 & -1 \\
1 & 1 & 0 & 0 & 0 & 0 & 1 & 1 & 0 \\
\hline 0 & 1 & 1 & 0 & 1 & 1 & 0 & 0 & 0 \\
1 & 0 & 1 & 1 & 0 & 1 & 0 & 0 & 0 \\
-1 & 1 & 0 & 1 & -1 & 0 & 0 & 0 & 0
\end{array}\right) .
$$

\subsection{Determination of S-matrix}

In an interval $\left[R_{I}, R_{O}\right]$ outside the range of the potential, the wave function has the form

$$
\psi_{\beta+(\alpha-1) n}^{\prime}(r)=A_{\alpha \beta} h_{l_{\alpha}}^{-}(p r)+B_{\alpha \beta} h_{l_{\alpha}}^{+}(p r)
$$

with the spherical Hankel functions $h_{l}^{ \pm}(p r)$ depending on the CMS momentum $p$. After determining the coefficients $A_{\alpha \beta}$ and $B_{\alpha \beta}$ from a fit to the wave function in this interval, the S-matrix can be constructed as

$$
S=\left(\begin{array}{ccc}
B_{11} & \cdots & B_{1 n} \\
\vdots & \ddots & \vdots \\
B_{n 1} & \cdots & B_{n n}
\end{array}\right)\left(\begin{array}{ccc}
A_{11} & \cdots & A_{1 n} \\
\vdots & \ddots & \vdots \\
A_{n 1} & \cdots & A_{n n}
\end{array}\right)^{-1}
$$


Without the mixing potential, the second matrix in this equation would have zero determinant and could not be inverted. For plotting, the S-matrix is decomposed according to the BlattBiedenharn parametrization [24]

$$
S=O^{-1} \operatorname{diag}\left(e^{2 i \delta_{1}}, \ldots, e^{2 i \delta_{n}}\right) O, \quad O^{T}=O^{-1},
$$

into the $n$ phase shifts $\delta_{1}, \ldots, \delta_{n}$ and the $n(n-1) / 2$ mixing angles

$$
\epsilon_{\alpha \beta}=\tan ^{-1} O_{\alpha \beta},
$$

for $\alpha, \beta=1, \ldots, n$ and $\beta>\alpha$.

\section{Computational results}

We consider the coupled ${ }^{1} D_{2} /{ }^{5} S D G_{2}$-wave for the test potential in Eq. (5) as a representative example. The lattice parameters used here have been partly adopted from Ref. [19]:

$$
\begin{aligned}
& a=1.97 \mathrm{fm}, \quad L=35 a, \quad R_{I}=9.02 a, \quad R_{O}=12.02 a, \\
& R_{W}=15.02 a, \quad \Lambda=10^{8} \mathrm{MeV}, \quad U_{0}=5 \mathrm{MeV} .
\end{aligned}
$$

Fig. 1 shows the obtained phase shifts and mixing angles as functions of the CMS momentum $p$. Obviously, the lattice results agree with a continuum calculation performed for comparison up to $p=120 \mathrm{MeV}$. (More precisely, the phase shifts and mixing angles on the lattice deviate from the continuum data by less than $0.5 \mathrm{deg}$ in the considered momentum interval.)

\section{Conclusion}

In this article, the lattice method from Ref. [19] has been generalized to an arbitrary number of coupled scattering channels. The generalized method has been benchmarked using the system of two spin-1 bosons interacting with a toy-model potential, for which the lattice and continuum results agree up to CMS momenta well below the lattice cutoff $\Lambda_{\text {latt }} \sim \pi / a \simeq 314 \mathrm{MeV}$ employed here. However, the presented technique can also be applied to particles with different spin combinations by modifying the radial states on the lattice. Moreover, it can be combined with the adiabatic projection method in order to consider scattering of particle clusters. Together with the chiral EFT interactions, this should allow to compute $n$-channel nuclear reactions on the lattice (e.g. deuteron-deuteron or deuteron-alpha scattering). 

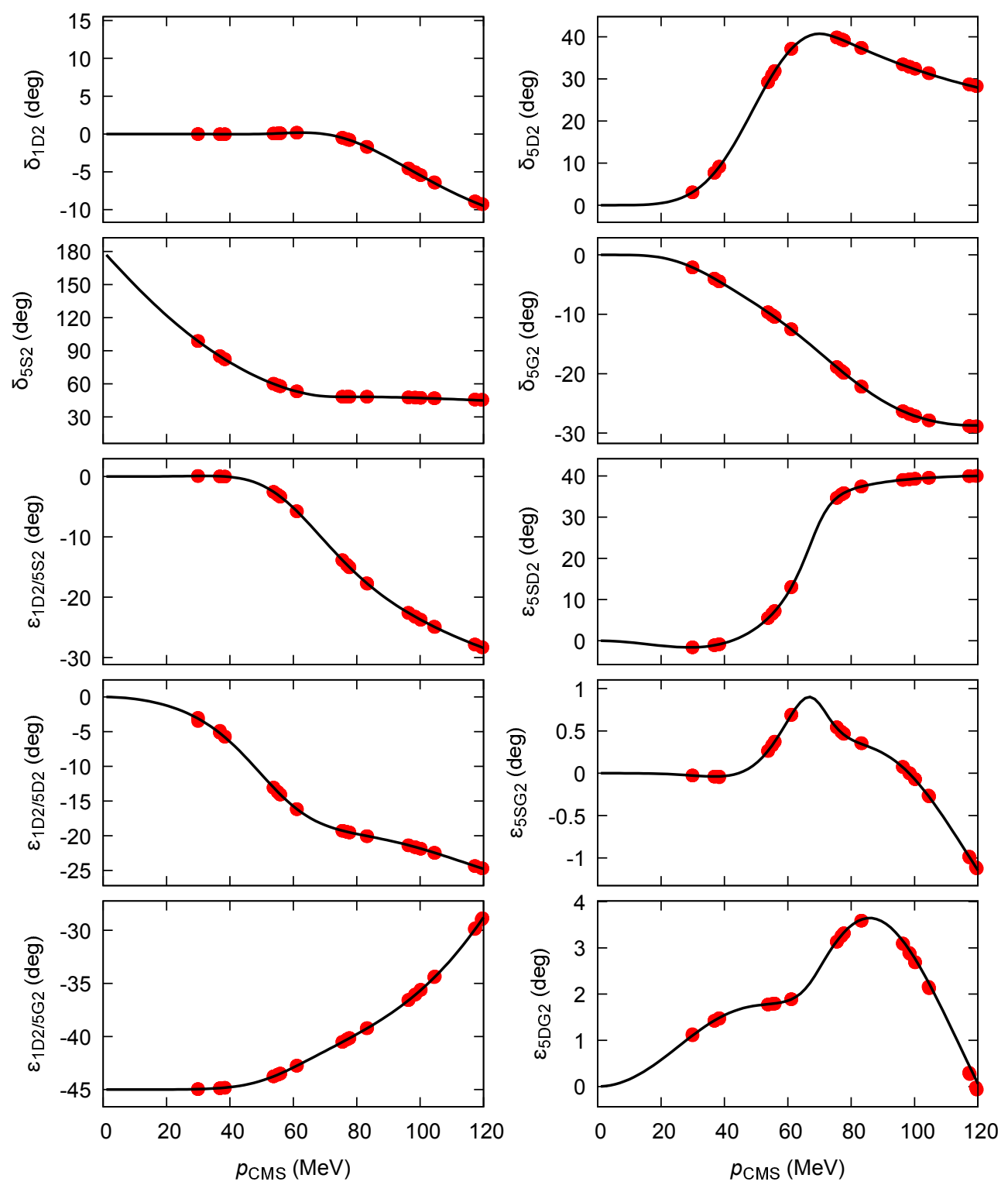

Figure 1: Phase shifts and mixing angles for the ${ }^{1} D_{2} /{ }^{5} S D G_{2}$-wave (black solid line: continuum; red points: lattice).

\section{Acknowledgements}

We are grateful to Ning Li and Bing-Nan Lu for helpful discussions and to Ulf-G. Meißner and Xiu-Lei Ren for sharing their insights into the discussed topics. We also thank Ning Li for sharing his nucleon-nucleon scattering code.

Funding information This work was supported by DFG (SFB/TR 110, "Symmetries and the Emergence of Structure in QCD"), the BMBF (Grant No. 05P2015) and the U.S. Department of Energy (DE-SC0018638 and DE-AC52-06NA25396). 


\section{References}

[1] M. Tanabashi et al., Review of particle physics, Phys. Rev. D 98, 030001 (2018), doi:10.1103/PhysRevD.98.030001.

[2] M. Lüscher, Volume dependence of the energy spectrum in massive quantum field theories. I. Stable particle states, Commun. Math. Phys. 104, 177 (1986), doi:10.1007/BF01211589.

[3] M.Lüscher, Volume dependence of the energy spectrum in massive quantum field theories. II. Scattering states, Commun. Math. Phys. 105, 153 (1986), doi:10.1007/BF01211097.

[4] M. Lüscher, Two-particle states on a torus and their relation to the scattering matrix, Nucl. Phys. B 354, 531 (1991), doi:10.1016/0550-3213(91)90366-6.

[5] R. A. Briceño, Two-particle multichannel systems in a finite volume with arbitrary spin, Phys. Rev. D 89, 074507 (2014), doi:10.1103/PhysRevD.89.074507.

[6] G. Moir, M. Peardon, S. M. Ryan, C. E. Thomas and D. J. Wilson, Coupled-channel $D \pi, D \eta$ and $D_{s} \bar{K}$ scattering from lattice $Q C D$, J. High Energ. Phys. 10, 011 (2016), doi:10.1007/JHEP10(2016)011.

[7] R. A. Briceño, J. J. Dudek, R. G. Edwards and D. J. Wilson, Isoscalar $\pi \pi, K \bar{K}, \eta \eta$ scattering and the $\sigma, f_{0}, f_{2}$ mesons from $Q C D$, Phys. Rev. D 97, 054513 (2018), doi:10.1103/PhysRevD.97.054513.

[8] A. Woss, C. E. Thomas, J. J. Dudek, R. G. Edwards and D. J. Wilson, Dynamically-coupled partial-waves in $\rho \pi$ isospin-2 scattering from lattice $Q C D$, J. High Energ. Phys. 07, 043 (2018), doi:10.1007/JHEP07(2018)043.

[9] A. J. Woss, C. E. Thomas, J. J. Dudek, R. G. Edwards and D. J. Wilson, $b_{1}$ resonance in coupled $\pi \omega, \pi \phi$ scattering from lattice QCD, Phys. Rev. D 100, 054506 (2019), doi:10.1103/PhysRevD.100.054506.

[10] E. Epelbaum, H. Krebs, D. Lee and U. -G. Meißner, Lattice chiral effective field theory with three-body interactions at next-to-next-to-leading order, Eur. Phys. J. A 41, 125 (2009), doi:10.1140/epja/i2009-10764-y.

[11] E. Epelbaum, H. Krebs, D. Lee and U.-G. Meißner, Ab Initio calculation of the Hoyle state, Phys. Rev. Lett. 106, 192501 (2011), doi:10.1103/PhysRevLett.106.192501.

[12] E. Epelbaum, H. Krebs, T. A. Lähde, D. Lee and U.-G. Meißner, Structure and rotations of the Hoyle state, Phys. Rev. Lett. 109, 252501 (2012), doi:10.1103/PhysRevLett.109.252501.

[13] E. Epelbaum, H. Krebs, T. A. Lähde, D. Lee, U.-G. Meißner and G. Rupak, Ab Initio calculation of the spectrum and structure of ${ }^{16} O$, Phys. Rev. Lett. 112, 102501 (2014), doi:10.1103/PhysRevLett.112.102501.

[14] T. A. Lähde, E. Epelbaum, H. Krebs, D. Lee, U.-G. Meißner and G. Rupak, Lattice effective field theory for medium-mass nuclei, Phys. Lett. B 732, 110 (2014), doi:10.1016/j.physletb.2014.03.023.

[15] S. Elhatisari, D. Lee, G. Rupak, E. Epelbaum, H. Krebs, T. A. Lähde, T. Luu and U.-G. Meißner, Ab initio alpha-alpha scattering, Nature 528, 111 (2015), doi:10.1038/nature16067. 
[16] S. Elhatisari, D. Lee, U.-G. Meißner and G. Rupak, Nucleon-deuteron scattering using the adiabatic projection method, Eur. Phys. J. A 52, 174 (2016), doi:10.1140/epja/i201616174-2.

[17] J. M. Alarcón, D. Du, N. Klein, T. A. Lähde, D. Lee, N. Li, B.-N. Lu, T. Luu and U.-G. Meißner, Neutron-proton scattering at next-to-next-to-leading order in Nuclear Lattice Effective Field Theory, Eur. Phys. J. A 53, 83 (2017), doi:10.1140/epja/i2017-12273-x.

[18] N. Li, S. Elhatisari, E. Epelbaum, D. Lee, B.-N. Lu and U.-G. Meißner, Neutron-proton scattering with lattice chiral effective field theory at next-to-next-to-next-to-leading order, Phys. Rev. C 98, 044002 (2018), doi:10.1103/PhysRevC.98.044002.

[19] B.-N. Lu, T. A. Lähde, D. Lee and U.-G. Meißner, Precise determination of lattice phase shifts and mixing angles, Phys. Lett. B 760, 309 (2016), doi:10.1016/j.physletb.2016.06.081.

[20] A. Rokash, M. Pine, S. Elhatisari, D. Lee, E. Epelbaum and H. Krebs, Scattering cluster wave functions on the lattice using the adiabatic projection method, Phys. Rev. C 92, 054612 (2015), doi:10.1103/PhysRevC.92.054612.

[21] J. Carlson, V. R. Pandharipande and R. B. Wiringa, Variational calculations of resonant states in ${ }^{4} \mathrm{He}$, Nucl. Phys. A 424, 47 (1984), doi:10.1016/0375-9474(84)90127-1.

[22] B. Borasoy, E. Epelbaum, H. Krebs, D. Lee and U. -G. Meißner, Two-particle scattering on the lattice: Phase shifts, spin-orbit coupling, and mixing angles, Eur. Phys. J. A 34, 185 (2007), doi:10.1140/epja/i2007-10500-9.

[23] L. Bovermann, E. Epelbaum, H. Krebs and D. Lee, Scattering phase shifts and mixing angles for an arbitrary number of coupled channels on the lattice, Phys. Rev. C 100, 064001 (2019), doi:10.1103/PhysRevC.100.064001.

[24] J. M. Blatt and L. C. Biedenharn, Neutron-proton scattering with spin-orbit coupling. I. General expressions, Phys. Rev. 86, 399 (1952), doi:10.1103/PhysRev.86.399. 